\title{
Photonic Crystal Microcavities for Classical and Quantum Information Processing
}

\author{
Jelena Vuckovic, Hatice Altug, Dirk Englund, Andrei Faraon, Ilya Fushman, Edo Waks \\ Department of Electrical Engineering and Ginzton Laboratory,Stanford University, Stanford, CA 94305-4088 \\ http://www.stanford.edu/group/nqp, jela@stanford.edu
}

\section{ABSTRACT}

Photonic crystal (PC) cavities enable localization of light into volumes $(V$ ) below a cubic optical wavelength (smaller than any other types of optical resonators) with high quality $(Q)$ factors [1]. This permits a strong interaction of light and matter, which is relevant for construction of classical light sources with improved properties (e.g., low threshold lasers) and of nonclassical light sources (such as single and entangled photon sources), which are crucial pieces of hardware of quantum information processing systems. This talk will cover some of our recent experimental results on quantum and classical devices enabled by such interaction [2-4], as well as our work on designing such devices and circuits efficiently [5-7].

We have demonstrated a spontaneous emission rate enhancement by a factor of 8 and suppression by a factor of 5 for a single self-assembled InAs/GaAs quantum dot (QD) embedded in a GaAs photonic crystal cavity and on- and off-resonance with the cavity mode, respectively [2]. A strong localization of optical field in such a nanocavity (experimental $Q$-factor of 5000 and mode volume below a cubic optical wavelength) with a quantum dot embedded inside is of importance for building single photon sources with improved efficiency, photon indistinguishability, and repetition rate. We have demonstrated a single photon source on demand based on the pulsed excitation of a single quantum dot in such a nanocavity, with pulse duration between 200 ps and $8 \mathrm{~ns}$ and with a small multi-photon probability (as small as 5\% compared to an attenuated laser of the same intensity) [2]. In addition, we have shown that colloidal PbS quantum dots coupled to AlGaAs photonic crystal cavities can be used as an alternative to self-assembled InAs/GaAs quantum dots for construction of cheap and reusable quantum and classical light emitters [3].

We have also demonstrated an improved classical light source - laser, based on coupling of a large number (81) of photonic crystal nanocavities inside a two dimensional array. Such a laser exhibits a low lasing threshold $(\sim 2.5 \mathrm{~mW})$, operates in a single mode, produces large output powers (greater than $12 \mu \mathrm{W}$, which two orders of magnitude larger than a single nanocavity laser), and can be directly modulated as speeds greater than $100 \mathrm{GHz}[4]$.

An inverse problem approach to designing photonic crystal cavities that we have developed enables their rapid optimization in a single step, thereby reducing the cavity optimization time from weeks to hours [5]. We are also pursuing theoretical and experimental work on integration of a number of photonic crystal components (cavities and waveguides) into functional circuits for classical and quantum information processing, including nontrivial two-qubit quantum gates [6-7].

\section{ACKNOWLEDGMENT}

This work has been supported by the ARO-DTO MURI Center for Photonic Quantum Information Systems, MARCO IFC, NSF and DARPA.
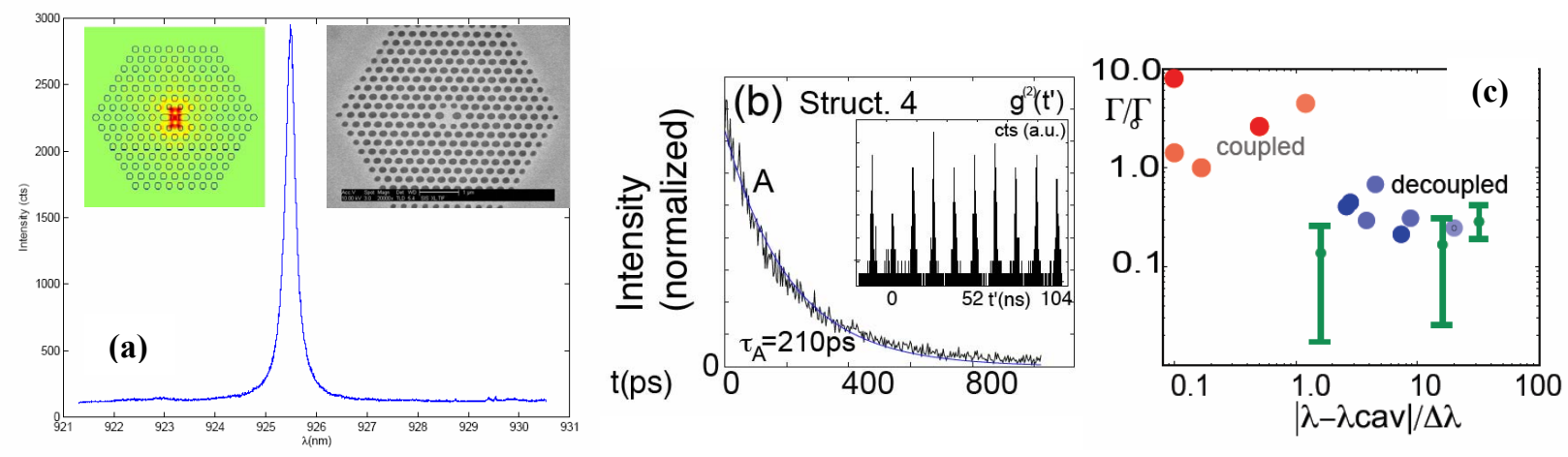

Fig. 1. (a) GaAs PC cavity with embedded InAs QDs (right inset; scalebar is $1 \mu \mathrm{m}$ ) supports the dipole mode (left inset) with small mode volume $V \sim 1 / 2(\lambda n)^{3}$ and $Q$-factor as high as $Q \sim 5000$ (central panel). (b) Measured radiative lifetime reduction for a single $Q D$ embedded in such a cavity; the lifetime is reduced from $\sim 1.7 \mathrm{~ns}$ to $\sim 200 \mathrm{ps}$. The inset shows the measured $2^{\text {nd }}$ order correlation function $g^{(2)}(t)$ for the same $Q D$, indicating the suppression of the multiphoton probability to $16 \%$ relative to an attenuated laser of the same intensity. (c) Experimental results for the spontaneous emission rate modification of a single InAs/GaAs $Q D$ embedded in such a cavity, as a function of its spectral detuning from the cavity resonance. Up to 8fold enhancement and 5-fold suppression of spontaneous emission rate relative to its rate without the cavity are observed [2]. 

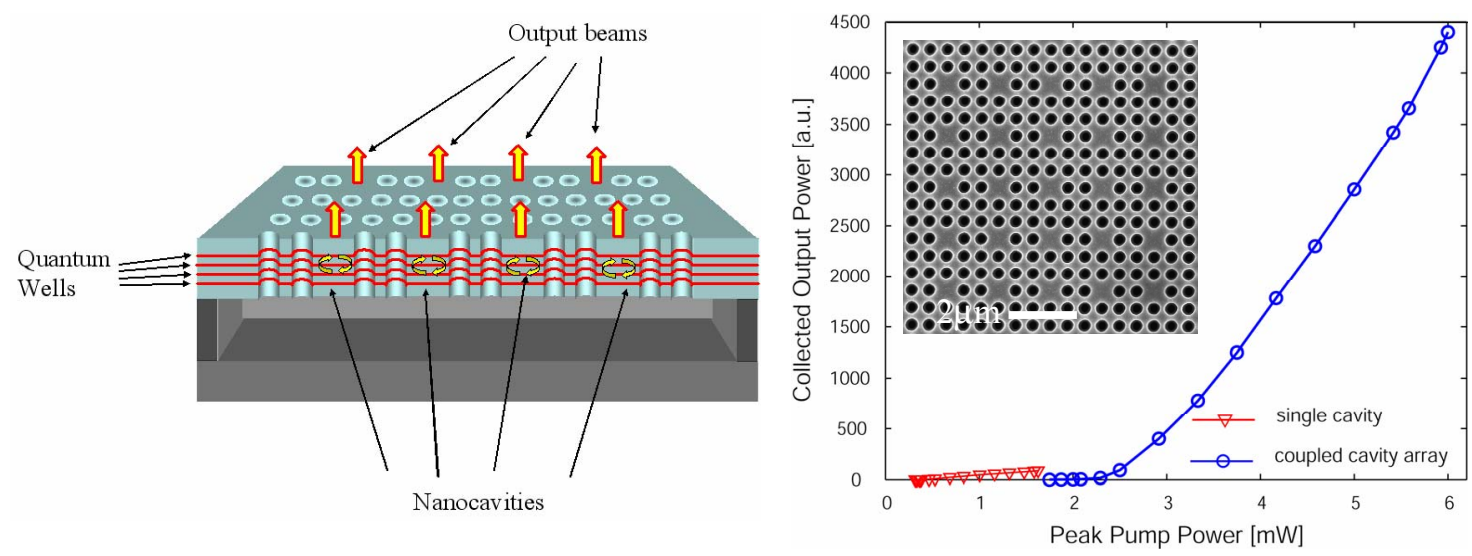

Fig. 2. Left: Photonic crystal nanocavity array laser design. Right: The output vs. input power curves for such a laser (red) and a single nanocavity laser (blue). Output powers from nanocavity array (shown in the inset) are more than two orders of magnitude larger, while threshold power increases by only a factor of 10 [4].

\section{REFERENCES:}

[1] K. Vahala, Optical microcavities, Nature, vol. 424, pp. 839-846 (2003).

[2] D. Englund, D. Fattal, E. Waks, G. Solomon, B. Zhang, T. Nakaoka, Y. Arakawa, Y. Yamamoto, and J. Vuckovic, Controlling the Spontaneous Emission Rate of Single Quantum Dots in a 2D Photonic Crystal, Physical Review Letters, vol. 95, 013904 (2005).

[3] I. Fushman, D. Englund, and J. Vuckovic, Coupling of PbS quantum dots to photonic crystal cavities at room temperature, Applied Physics Letters, vol. 87, 241102 (2005).

[4] H. Altug and J. Vuckovic, Photonic crystal nanocavity array laser, Optics Express, vol. 13, no. 22, pp. 8819-8828 (Nov. 2005).

[5] D. Englund, I. Fushman, and J. Vuckovic, General recipe for designing photonic crystal cavities, Optics Express, vol. 13, no. 16, pp. 5961-5975 (2005).

[6] E. Waks and J. Vuckovic, "Coupled mode theory for photonic crystal cavity-waveguide interaction," Optics Express, vol.13, no. 13, pp. 5064-5073 (2005).

[7] E. Waks and J. Vuckovic, Dipole Induced Transparency in drop filter cavity-waveguide systems, Physical Review Letters, vol. 96, article 153601 (2006). 